\title{
Restrictive Effect Analysis of Context on the Sentences Choice in the Perspective of Contemporary Pragmatics
}

\author{
Jie Bai \\ School of Humanities, Xi'an Peihua University, Xi'an 710125, China
}

\begin{abstract}
There is a very close relationship between context and sentence choice, the specific context must be matched with the language form. This paper focuses on the context of the functional point of view to explore the role of context on constraints of the sentence choice from the perspective of pragmatic language.

Keywords: restrictive effect analysis, context, sentences choice, contemporary pragmatics
\end{abstract}

\section{Introduction}

In recent decades, due to linguistics, especially the emergence and development of pragmatics, the study of context has penetrated into all areas of linguistics, and is still in-depth and development, the language has become the linguistic circles Concerned about a topic. But what is the context, due to different disciplines, different schools of the context of the analysis of the point of view, different points of view and the answer is not exactly the same. Until now, its interpretation is still very broad, not strictly limited, arbitrariness. As early as the early 1920s, Polish scholar Malinowski proposed the influence and restraint of "cultural context" and "contextual context" on discourse meaning. He believes that language is the behaviour, not the ideological signal, discourse and the environment closely together, the language environment is essential to understand the language. In the 1930s, the English linguist Firth made a further elaboration on the context and put forward the context theory. He believes that not only the sentence of the sentence and the next sentence, the upper or lower 
paragraphs of a paragraph is "context" and language and social environment is the relationship between "context" and the former is the context of language factors, which is the context. Forth did not simply think that only when the sentence into the communicative field, only with the context of the relationship between the language factors he took into account, which is a major progress in contextual research. 60 s of last century, the system functional grammar Halliday put forward the concept of register. He divided the complex context factors into three categories: the scope of discourse, discourse and discourse tone.

Chen Wangdao, the founder of Chinese rhetoric, has discussed the necessity and significance of context to rhetoric in the light of Marxist linguistic notions and the "rhetoric to adapt to the situation as the first meaning of the title" of the famous assertion linguistic circles. Since the 1960s, the domestic research on the context of a wide range. There are three different ways to divide into three categories: the first is based on the pragmatic point of view, which is based on the subject of language act. It clearly states that "context is the language environment, The second is based on the reality of verbal communication, the context is divided into "real context" and "unrealistic context (generalized context)"; the third from the language and verbal communication, the context is divided into "Language environment", "non-language environment"; "verbal context, verbal context". The terms of these components are different, but the content is roughly the same.

From the above results, we can see that context not only contains the language knowledge shared by both sides of the communication, that is, syntactic rules, vocabulary, phonological rules and so on, as well as the context of the language, but also includes Extra-linguistic knowledge, that is, knowledge related to a particular communicative context and background knowledge beyond a particular communicative context. Therefore, this paper argues that context is the language environment in which a particular meaning is expressed. It includes the language environment and language environment. Language context mainly refers to "context" or "preface"; language environment is mainly situational knowledge, background knowledge, communication between the two sides of mutual knowledge. The role of context in language communication is multifaceted, and now it is a little understanding of its control function.

\section{The pragmatic study}

Pragmatics is the study of the use and understanding of language learning, both the study of the speaker using language and external context to express the meaning of the process, but also study the speaker to the speaker to speak the discourse of the decoding and reasoning process. As with semantics, pragmatics also deals with meaning, but it does not study the meaning of the abstract linguistic system itself, but the meaning communicated and understood by the communicator in a given communicative context and the process of understanding and communicating. Translation studies are to explore the translator to interpret the original, in the translation of the original meaning of the 
reconstruction of knowledge. The two have a common object of study, namely, language understanding and language expression. The former are more concerned with oral communication and its dynamic characteristics, while the latter is concerned with text and written language. But this difference does not prevent the translation theory researchers from the use of pragmatic theory to explore translation activities. In fact, many scholars believe that written discourse, like verbal discourse, has a communicative nature and characteristics and is dynamic to express their intentions to the reader by means of the discourse (works), which they consider themselves to be in writing. If the author believes that the reader is not familiar with a certain knowledge or mood and will affect the reader's interpretation of the work, understanding, he will write this detail, and vice versa. By reading the author's works, the reader can acquire new knowledge, fresh feelings, experience, resonate with the author, and even take some action by the influence of the works, successfully completing the author-reader communication.

\section{The contextual constraints}

The restriction function of context refers to the limitation of context to language expression. Constraint function is for the writer, speaking and writing language to express thoughts and feelings, in order to express accurate and clear, concise and vivid, in addition to the rules of expression in accordance with the language of expression, but also pay attention to language structure adjustment and Select, pay attention to the use of some clever and purpose of the special expression, so that listeners to resonate, happy to accept. How to adjust and select the language material is determined by the specific language environment, only to meet the specific context to ensure the smooth transmission of discourse information, to obtain the best expression effect.

\section{Research on the constraints role of context on sentence choice}

Language environment refers to the people in the language and the state of the situation in public relations linguistics language environment mainly refers to the language activities of people when the time, place, occasions and other factors, including understanding the preamble to help analyze semantics. The subjective factors include the identity of the language communicator, ideas, disposition, character training, mood and so on. The use of language includes listening, speaking, reading, writing four aspects. Listening and reading is the process of understanding the language is to receive information sharp, written language is the process of sending information. Understanding language and language expression are different stages of the communication process, but are subject to the language environment.

The so-called sentence means that a sentence must be in accordance with a certain pattern to organize. Such as arranging sentence imperative sentence. 
There are passive sentence, predicate sentence, predicate object, attributive postposition, adverbial postposition, subject postposition, ingredient omission sentence, preposition object in advance, interrogative clause pronoun object predicate and negative pronoun object. Context is an important area of linguistic research. There are two kinds of influence of language environment on sentence structure. One is restriction and the other is supplement. This paper mainly analyzes the limitation effect. Sentence is the mode of language organization when language is communicated, so the impact of language environment on sentence structure is easier to show its restrictive effect from understanding and expression.

First of all, as the understanding of part of the listening and reading will be extended to the language of speech and text, because the words exist homophone, the word and other situations when the need for language communication before and after the auxiliary language to understand; as part of the expression. Writing and listening and reading have a similar role in the expression itself is to let each other understand the purpose.

Now the academic community for the nature of the language environment is also different opinions, but there are two main points of view. One is the context of the narrow sense that the language environment is an attribute of language, so the context must be limited to the language that is to focus on the context of context. The other is that the context of generalized has gone beyond the language category of the attribute must be on the language. And verbal communication-related factors to its qualitative. A specific locale must have a language form that matches it. The relationship between language environment and sentence choice is very close. The language environment restricts the choice of sentence pattern to match the context on the other hand and the sentence must be able to express the specific context meaning.

The choice of sentence structure is the choice of synonymous sentences. There is only a slight difference in the sentences with the same semantics and different sentences, which must be supplemented by context. This is not because of the contextual constraints, because the sentence arrangement is arranged according to a certain special style and then the language under this style has its special role in communication, making the listener in the context of the language environment to help more easily accept and understand its specific meaning.

Language environment on the use of language constraints also reflected in the understanding and organization of the sentence. The general sentence is easy to understand but some special sentences, leaving a certain context will be difficult to understand. From the above mentioned time, place, occasion, as well as the identity of the language of communication status, ideas, disposition, character training, mood, etc. to analyze the context of the sentence for the impact. To listen to the reader willing to accept, resonate, that the writer should not only enrich the content, but also with the perfect form of language. The language as the transmission of information exchange ideas and feelings of an important tool to improve its expression of the effect, so rich in sound and sound can not be separated from the match. Words with the sound well, read fluently, sounds sweet, easy to remember, it will help the expression of meaning, the transmission of 
information. But the cooperation of these voices and the results obtained by the context of constraints.

Speak, write the article to have "the same thing, 100 kinds of that" awareness, to stress the transformation of the sentence, to select the ability to train different sentences. The same meaning, you can use a variety of sentences to express, so the use of specific language, people in order to better express feelings, transmission of information, it is necessary according to the context and requirements, the appropriate sentence changes, so as to achieve good expression.

Through the above simple analysis of the context control function, it is said that the writer is speaking and writing, in order to make the reader understand it, from every stage of his determination of motivation to the determination of language content and form, and listening to the reader to receive speech information Context Restriction. Therefore, the context of participation is an essential element of any successful communication. Language out of a specific context, as if the fish left the water, they lost their lives. To sum up, we should pay full attention to the context of the constraints.

\section{Conclusion}

Language is an art, while language is also one of the most common social phenomena and interpersonal activities, so the language can not be separated from the specific environment. We learn the language not only as much as possible to learn voice, grammar and other related knowledge of the language, but also master the pronunciation and the meaning of the word to accurately determine the language. The appropriate use of the language in the language environment enhances the ability to actually use the language.

\section{References}

[1] Liu Yujuan. On the context of the constraints and explanatory functions]. Harbin Teachers College, 55(12), pp.68-70, 2011

[2] Han Caiying. Contextual Constraints and Their Expressions. Journal of Language and Literature, 8 (5), pp.87- 91, 2013

[3] Wang Huiqin. Analysis of the role of context in the use of language. Pingdingshan Teachers College, 12(10), pp. 18- 20, 2012.

[4] He Fengxian. On the relationship between context and meaning. Gansu Social Sciences, 9(6), pp.58- 61, 2011

[5] Huang Bairong, Liao Xudong. Modern Chinese. Beijing: Higher Education Press, pp.85-87, 2014 